\title{
Early Metabolic Response as a Predictor of Treatment Outcome in Patients With Metastatic Soft Tissue Sarcomas
}

\author{
MYRELLA VLENTERIE ${ }^{1}$, WIM JG OYEN ${ }^{2}$, NEELTJE STEEGHS ${ }^{3}$, INGRID M.E. DESAR ${ }^{1}$, \\ REMY B. VERHEIJEN ${ }^{4}$, ANNE MIEK KOENEN ${ }^{3}$, WILLEM GROOTJANS 5 , \\ LIOE-FEE DE GEUS-OEI ${ }^{2,5}$, NIELKA P. VAN ERP ${ }^{6 *}$ and WINETTE TA VAN DER GRAAF ${ }^{1,3 *}$ \\ ${ }^{1}$ Department of Medical Oncology, Radboud University Medical Center, Nijmegen, the Netherlands; \\ ${ }^{2}$ Department of Radiology and Nuclear Medicine, Radboud University Medical Center, Nijmegen, the Netherlands; \\ ${ }^{3}$ Department of Medical Oncology, Antoni van Leeuwenhoek - \\ Netherlands Cancer Institute, Amsterdam, the Netherlands; \\ ${ }^{4}$ Department of Pharmacy, Antoni van Leeuwenhoek - Netherlands Cancer Institute, Amsterdam, the Netherlands; \\ ${ }^{5}$ Department of Radiology, Leiden University Medical Center, Leiden, the Netherlands; \\ ${ }^{6}$ Department of Pharmacy, Radboud University Medical Center, Nijmegen, the Netherlands
}

\begin{abstract}
Background/Aim: Pazopanib is approved for advanced soft tissue sarcoma (STS) patients. The aim of the study was to examine the usefulness of $\left({ }^{18} \mathrm{~F}\right)$ Fluorodeoxyglucose-positron emission tomography/ computed tomography (FDG-PET/CT) imaging for early evaluation of the response of STS patients to pazopanib, as well as the association between pazopanib pharmacokinetics and early metabolic response. Patients and Methods: Twenty STS patients underwent FDG-PET scans at baseline, two- and eight-weeks following treatment with pazopanib. The FDGPET scans were evaluated by quantitative PERCIST analysis and visually by an independent nuclear medicine physician and related to RECIST1.1 outcome at eight weeks. Results: After eight weeks of therapy, 14 out of 20 patients had discontinued pazopanib due to tumor progression identified radiologically ('non-responders' $n=12$ ) or toxicity $(n=2)$. Quantitative FDG-PET scoring at two weeks, according to PERCIST guidelines, identified 25\% (3 of 12) of the patients radiologically as non-responders versus $42 \%$ (5 of 12) identified by visual response analysis. Conclusion: In this heterogeneous STS patients' cohort, early FDG-PET/CT identified a substantial part of pazopanib non-responders.
\end{abstract}

\footnotetext{
*These Authors contributed equally to this study.

Correspondence to: Myrella Vlenterie, Radboud University Medical Center, Geert Grooteplein Zuid 10, 6525 GA, Nijmegen, the Netherlands. Tel: +31 243610353, e-mail: myrella.vlenterie@ radboudumc.nl
}

Key Words: Sarcoma, soft tissue, positron-emission tomography, pharmacokinetics, pazopanib, pharmacodynamics.
Soft tissue sarcomas (STS) represent a group of rare mesenchymal cancers that encompasses more than 70 histological subtypes (1). Based on the outcome of the PALETTE trial, the Food and Drug Administration (FDA) and European Medicines Agency (EMA) approved pazopanib as the first oral targeted therapy for non-gastrointestinal stromal tumors (GIST), non-adipocytic STS patients in second line and beyond (2). Patients treated with pazopanib showed a progression-free survival (PFS) benefit of 3 months compared to placebo-treated patients. Unfortunately, $23 \%$ of patients had progressive disease and were considered as de novo resistant to pazopanib therapy (non-responders), and about $10-30 \%$ of the STS patients treated with pazopanib developed grade 3-4 toxicity $(2,3)$. It is, therefore, evident that easily assessable biomarkers to predict response shortly after the start of treatment with pazopanib are required to identify patients who will ultimately not benefit from therapy. This is particularly important to avoid treatment of patients with an ineffective, potentially toxic, and expensive drug over a prolonged period of time. Molecular imaging with $\left({ }^{18} \mathrm{~F}\right)$-FluorodeoxyglucosePositron Emission Tomography/Computed Tomography (FDG$\mathrm{PET} / \mathrm{CT}$ ) has shown clinical relevance for grading and staging sarcoma patients (4); reflecting outcome after (neoadjuvant) chemotherapy $(5,6)$; and for predicting the anti-tumor effect of imatinib in GIST patients $(7,8)$ at an earlier stage than is currently possible with conventional radiological imaging that solely depicts tumor morphology (CT/MRI). In addition, FDG$\mathrm{PET} / \mathrm{CT}$ has been successful in predicting the effects of angiogenesis inhibitors in metastatic renal cell carcinoma (mRCC) (9).

Until now, the metabolic response depicted by FDG-PET/CT has not been related to drug pharmacokinetics (PK), while drug concentration/exposure is recognized to be important for 
optimization of anti-tumor activity of several targeted therapies (10). Studies providing further insight into pharmacodynamic and pharmacokinetic biomarkers are essential to optimize pazopanib treatment for individual patients with STS and select those who are de novo resistant or sub-optimally treated. Although exposure to pazopanib might affect treatment outcome, as has been demonstrated for mRCC (11), this association is less clear for STS patients $(12,13)$.

The current study was designed to analyze the value of FDG-PET/CT imaging for early evaluation of the response of STS patients to pazopanib. In addition to early response monitoring with FDG-PET/CT, this study explored the association between pazopanib pharmacokinetics and early metabolic response.

\section{Patients and Methods}

Patients. Histologically diagnosed STS patients starting with pazopanib treatment were included in this study. Inclusion and exclusion criteria were similar to those used in the PALETTE trial (2). In addition, patients were required to have a positive baseline FDG-PET scan for continuation in the study and diabetic patients were only eligible if well controlled. The trial was approved by the regional medical ethics committee. All patients gave written informed consent. The study was registered at ClinicalTrials.gov (NCT01995981).

Study design. The study was designed as a prospective observational feasibility study. Since this was an exploratory feasibility study, the sample size of 20 was chosen. In this study two hypotheses were explored: 1. FDG-PET/CT can be used for early monitoring of response to pazopanib treatment in STS patients, and 2. there is an association between FDG-PET/CT response and pazopanib concentration/exposure. The study outline is illustrated in Figure 1A. All patients were instructed to take pazopanib during this study in a standardized manner: $800 \mathrm{mg}$ pazopanib once daily (OD), at 8:00 AM with a glass of water, one hour before having a standardized continental breakfast. Evaluation was performed at 2 weeks and 8 weeks after the start of treatment with pazopanib (the latter is at the time of conventional RECIST response evaluation). At both time points, blood samples were collected at $0,1,2,3,4$, $6,8,10$, and $24 \mathrm{~h}$ after ingestion of pazopanib and an FDG-PET/CT scan was performed. At eight weeks, a standard contrast-enhanced CT scan for tumor evaluation was incorporated in the FDG-PET/CT, and PFS was scored according to RECIST 1.1 (14) which is currently used as gold standard in clinical practice for pazopanib response evaluation in STS patients. Patients showing stable disease or response by RECIST were defined as responders to pazopanib, whereas patients showing progressive disease were defined as nonresponders. Additionally, adverse events during the study were graded by CTCAE v4.0 (15).

FDG-PET analysis. FDG-PET/CT-scans were acquired approximately one hour after injection of $1.6 \mathrm{MBq} / \mathrm{kg}$ FDG and patients that had been fasting for at least $6 \mathrm{~h}$ before injection according to the European Association of Nuclear Medicine (EANM) standardized guidelines (16). Whole body FDG-PET images were acquired using a Biograph $40 \mathrm{mCT}$ PET/CT scanner
(Radboud UMC: Siemens Healthcare, Knoxville, TN, USA; AVLNCI: Philips Medical Systems, GEMINI ToF Big Bore, Cleveland, OH, USA). The PET scanner was accredited by the research 4 life (EARL) initiative for quantitative multicenter PET/CT studies. PERCIST requirements in patient preparation, FDG-PET acquisition, evaluation and assessment were followed, which include obtaining the baseline PET scan at 50-70 min after tracer injection. To be able to compare FDG-PET scans of one patient, the time between tracer injection and scanning in the follow-up PET scans is not allowed to differ more than $15 \mathrm{~min}$ from the baseline PET scan. All scans had to be performed with the same injected dose $\pm 20 \%$ of FDG (17). FDG accumulation was registered in $\mathrm{Bq} / \mathrm{ml}$, which was then corrected for the injected dose, time elapsed between the injection and scanning, and gender specific lean body mass to obtain the standardized uptake value (SUL) (18). For the purpose of response monitoring, the peak standardized uptake value corrected for lean body mass ( $\mathrm{SUL}_{\text {peak) }}$ was determined in (a maximum of) five of the most metabolically active tumor lesions, with a maximum of two lesions per organ, on sequential FDGPET/CT images. The SUL $\mathrm{Seak}_{\text {pas }}$ calculated using a customized Matlab script (Matlab 2014b, Natick, MA, USA). This script automatically searched for the metabolically most active part of the tumor using a three-dimensional (3D) spherical volume of interest (VOI) of $1 \mathrm{ml}$. Metabolic changes were defined as a fractional change $\left(\Delta \mathrm{SUL}_{\text {peak }}\right)$, comparing the second or third scan with the baseline scan (Figure 2). As STS patients frequently have metastatic disease in the liver, uptake in the descending thoracic aorta was used as a control instead of uptake in normal liver. At baseline, no minimal uptake was required, as tumors were readily identifiable on FDG-PET/CT, showing FDG-uptake clearly above adjacent background levels. Adjacent lesions within a single organ (e.g. lymph node or bone lesions) that could not be measured separately were considered as one single target lesion. If necessary, adjacent normal tissue uptake in the bladder or myocardium was manually excluded from the VOI.

Quantitative metabolic partial response (qM-PR) was defined as $\geq 30 \%$ decrease in $\Delta \mathrm{SUL}_{\text {peak }}$, quantitative metabolic progressive disease (qM-PD) was defined as $\geq 20 \%$ increase in $\Delta S \mathrm{SL}_{\text {peak }}$ or new lesions according to PERCIST definitions (17). The remainder was defined as quantitative metabolically stable disease (qM-SD). Furthermore, the FDG-PET/CT images were visually scored in a blinded, independent central review by one experienced nuclear medicine physician. The categories of visual response assessment included metabolic partial response, stable disease, or progressive disease (visual-PR, $\mathrm{SD}$, and $\mathrm{PD}$, respectively). Additionally, quantitative metabolic response was analyzed per metastatic subtype tissue: lymph nodes, bone, abdominal organs, lung and others (consisting of subcutaneous tissue, muscle and perineum). If a patient had multiple lesions at one site, the average response for that metastatic site was calculated.

Pharmacokinetics. Blood samples were centrifuged at 3,000 $\times g$ for $5 \mathrm{~min}$ at room temperature; plasma was collected and split into two aliquots and stored at $-40^{\circ} \mathrm{C}$ until the day of analysis. Pazopanib plasma concentrations were determined using a validated ultraperformance liquid chromatography-tandem mass spectrometric method (LC-MS/MS) comparable to the earlier described bioanalytical method (19). The Area Under the Concentration Time Curve $\left(\mathrm{AUC}_{0-24 \mathrm{~h}}\right)$ for pazopanib was calculated using a noncompartmental trapezoidal approach (Phoenix WinNonlin v6.3 ${ }^{\circledR}$ ). 
A

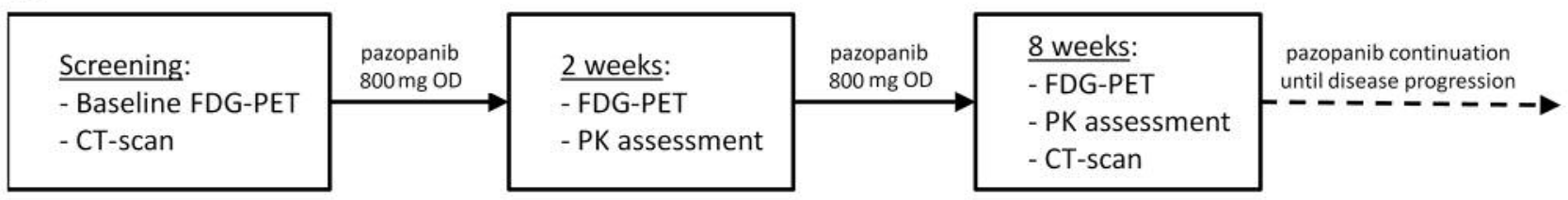

B

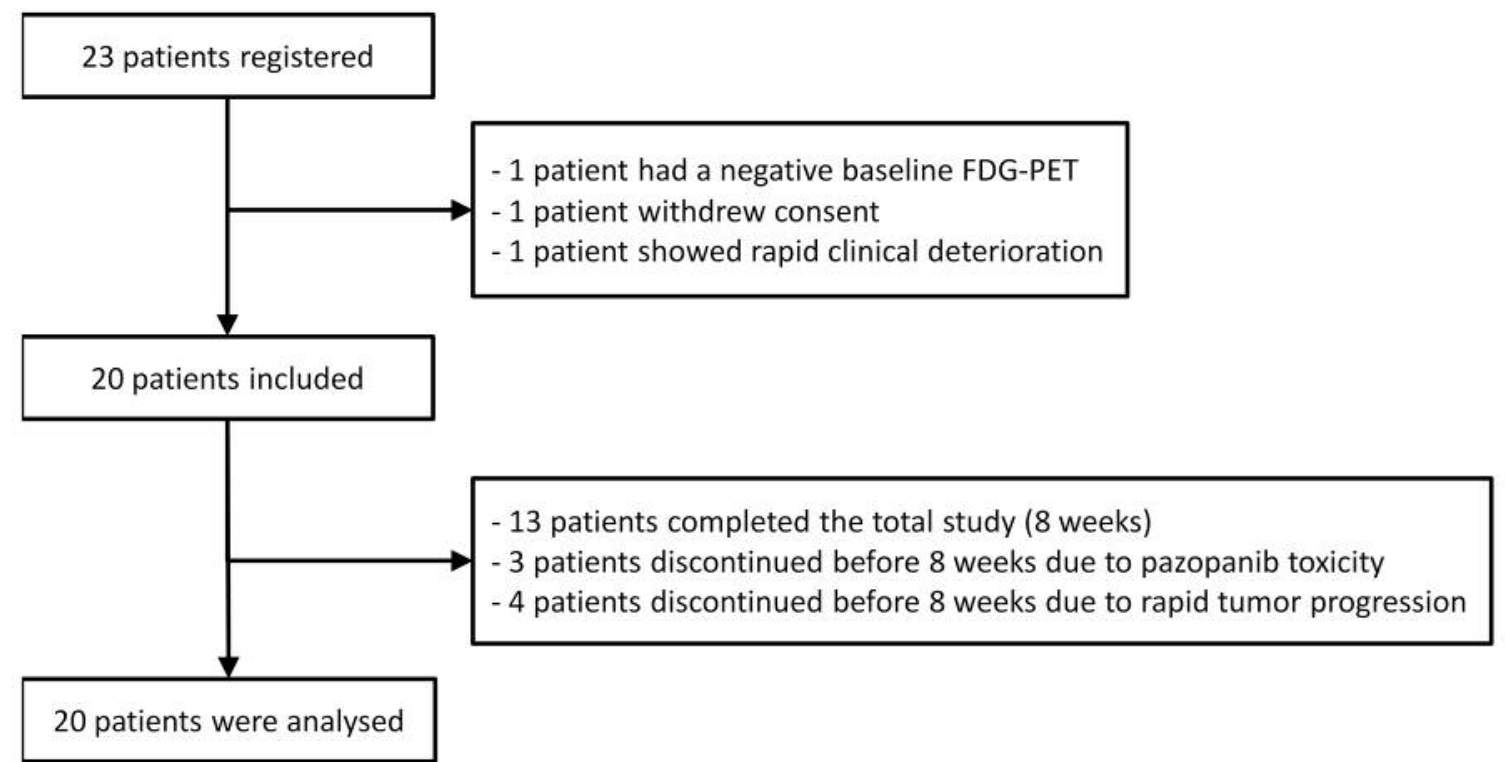

Figure 1. Study design. A: Study protocol timeline; a baseline FDG-PET/CT was performed 0-2 weeks before start of pazopanib therapy. B: study profile.

\section{Results}

Patient characteristics. Patients that were treated at two sites in the Netherlands (the Radboud University Medical Center in Nijmegen and the Antoni van Leeuwenhoek - Netherlands Cancer Institute in Amsterdam) between 2013-2017 were included in the study. The baseline patient characteristics are shown in Table I. A total of 23 patients were included in the study, of whom three dropped out before its start (Figure 1B). The remaining twenty patients had paired FDG-PET/CT scans at baseline and after two weeks of pazopanib treatment. Seven patients discontinued the study prematurely (before eight weeks of therapy) due to serious pazopanib toxicity $(n=3)$ or rapid tumor progression $(n=4)$, and therefore had no FDG-PET/CT and PK assessment after 8 weeks of treatment. Of the three patients that experienced serious toxicity, two discontinued pazopanib due to gastric bleeding $(n=1)$ or hepatotoxicity $(n=1)$. The third patient also developed hepatotoxicity but could continue pazopanib at a lower dosage $(600 \mathrm{mg})$ and was therefore included in the survival analyses of this study. After eight weeks of therapy (the time of first clinical evaluation) eight additional patients showed progressive disease on CT. Thus, a total of 12 patients $(60 \%)$ (excluding the two patients who stopped due to toxicity and therefore response remains unknown) discontinued pazopanib due to tumor progression, and were defined as 'non-responders'. The six patients (30\%) who experienced clinical benefit (all scored as stable disease by RECIST 1.1) continued pazopanib for 5-18 months until disease progression occurred. Patients who responded to pazopanib had a synovial sarcoma $(n=2)$, myxofibrosarcoma $(n=1)$, unclassified spindle cell sarcoma $(n=1)$, pleiomorphic undifferentiated high-grade sarcoma $(n=1)$ and epithelioid hemangioendothelioma $(n=1)$.

FDG-PET assessment. In total, 53 FDG-PET/CT scans were performed in 20 patients. Four scans (7.5\%) showed a variation of $>0.3$ SUL difference in the descending aorta, 


\section{A: Baseline FDG-PET/CT}

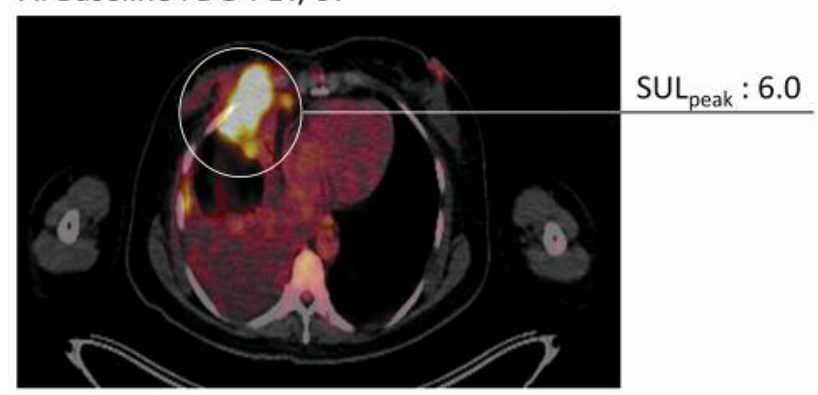

\section{B: FDG-PET/CT after two weeks}

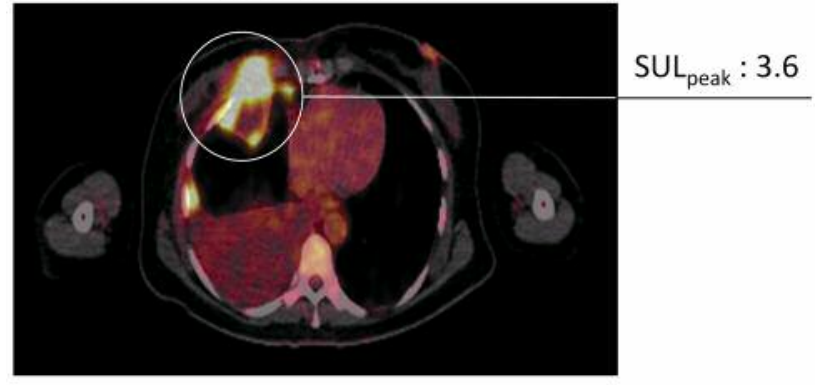

Figure 2. FDG-response monitoring.

which is considered undesirable when comparing two FDGPET/CT scans quantitatively (17). As this reflects clinical practice, all FDG-PET/CT were included in the analysis of the study. At two weeks, based on the PERCIST guidelines, four of the 18 patients showed a quantitative metabolic (qM)-PR, and three patients showed qM-PD (two due to metabolic increase $\geq 20 \%$, and one based on new lesions (in calculations scored as $+20 \%)$ ). The remaining 11 patients had qM-SD (Figure 3). In addition to the FDG-PET/CT at two weeks, an FDG-PET/CT was also performed at 8 weeks in combination with the $\mathrm{CT}$ for clinical evaluation (RECIST). Overall, 13 patients completed this FDG-PET/CT at eight weeks, of whom three (23\%) showed qM-PR and three $(23 \%)$ qM-PD. In these six patients, contrast-enhanced CT evaluation according to RECIST 1.1 corresponded with the FDG-PET/CT scan results and therefore, the patients continued and discontinued pazopanib accordingly. Of the seven patients with metabolically stable disease $(-13 \%$ to $+15 \%$ versus baseline) after eight weeks of therapy, five had radiological progressive disease and discontinued pazopanib while two had a clinical response and continued pazopanib.

Besides the quantitative PERCIST analysis, which is currently considered to be too time consuming for general clinical practice, an independent visual response analysis at two weeks (non-quantitative) was performed. This evaluation was executed by one experienced, independent nuclear
Table I. Baseline patient characteristics.

\begin{tabular}{|c|c|}
\hline Variable & Total $\mathrm{n}=20(\%)$ \\
\hline \multicolumn{2}{|l|}{ Age (yrs) } \\
\hline Range (yrs) & $40-78$ \\
\hline Median (yrs) & 60 \\
\hline Gender (male) & $9(45)$ \\
\hline \multicolumn{2}{|l|}{ STS subtypes } \\
\hline Leiomyosarcoma & $7(35)$ \\
\hline Synovial sarcoma & $2(10)$ \\
\hline Angiosarcoma & $2(10)$ \\
\hline Myxofibrosarcoma & $2(10)$ \\
\hline Pleiomorphic rhabdomyosarcoma & $1(5)$ \\
\hline Epithelioid hemangioendothelioma & $1(5)$ \\
\hline Solitary Fibrous tumor & $1(5)$ \\
\hline Unclassified spindle cell sarcoma & $1(5)$ \\
\hline Epithelioid sarcoma & $1(5)$ \\
\hline Desmoplastic small blue round cell tumor & $1(5)$ \\
\hline Pleiomorphic undifferentiated high grade sarcoma & $1(5)$ \\
\hline \multicolumn{2}{|l|}{ ECOG } \\
\hline 0 & $4(20)$ \\
\hline 1 & $13(65)$ \\
\hline 2 & $3(15)$ \\
\hline \multicolumn{2}{|l|}{ Duration of disease from diagnosis until pazopanib start } \\
\hline Range (months) & $1-248$ \\
\hline Lines of chemotherapy before pazopanib ${ }^{\mathrm{a}}$, range & $0-4$ \\
\hline \multicolumn{2}{|l|}{ Progressive disease at 8 weeks } \\
\hline Yes & $12(60)$ \\
\hline No & $6(30)$ \\
\hline Missing & $2(10)$ \\
\hline Progressive disease median (months) & 2 \\
\hline Progressive disease range (months) & $1-18$ \\
\hline Overall survival median (months) ${ }^{b}$ & 8 \\
\hline Overall survival range (months) & $1-24$ \\
\hline Maximum uptake at baseline $\left(\mathrm{SUL}_{\text {peak }}\right)$ & $1.92-24.40$ \\
\hline
\end{tabular}

aThe majority of patients had received one line of chemotherapy prior to pazopanib treatment (11 of 20 patients, $55 \%$ ). Of these, six had received doxorubicin and three the combination of doxorubicin and ifosfamide; bThree patients still alive at study closure, and one patient's OS data are unknown; 'The large variation in maximum uptake at baseline reflects the metabolic heterogeneity of the sarcoma subtypes.

medicine physician who was blinded to all other clinical data. FDG-PET/CT scans were scored as visual-PR $(n=9)$, visual-SD $(n=4)$, or visual-PD $(n=5)$. All patients with FDG$\mathrm{PET} / \mathrm{CT}$ scans scored as visual-PD at two weeks showed radiological progressive disease at eight weeks and discontinued pazopanib. Therefore, at two weeks, visual scoring identified more non-responders (42\%) than the quantitative scoring $(25 \%)$. The calculated sensitivity and specificity for disease progression by visual response was $42 \%$ and $100 \%$ respectively, in comparison to $25 \%$ and $100 \%$ by quantitative analysis.

Of note, it is important to be aware of FDG response differences between the various tissues. Although the group size was small, bone lesions in general showed an early 


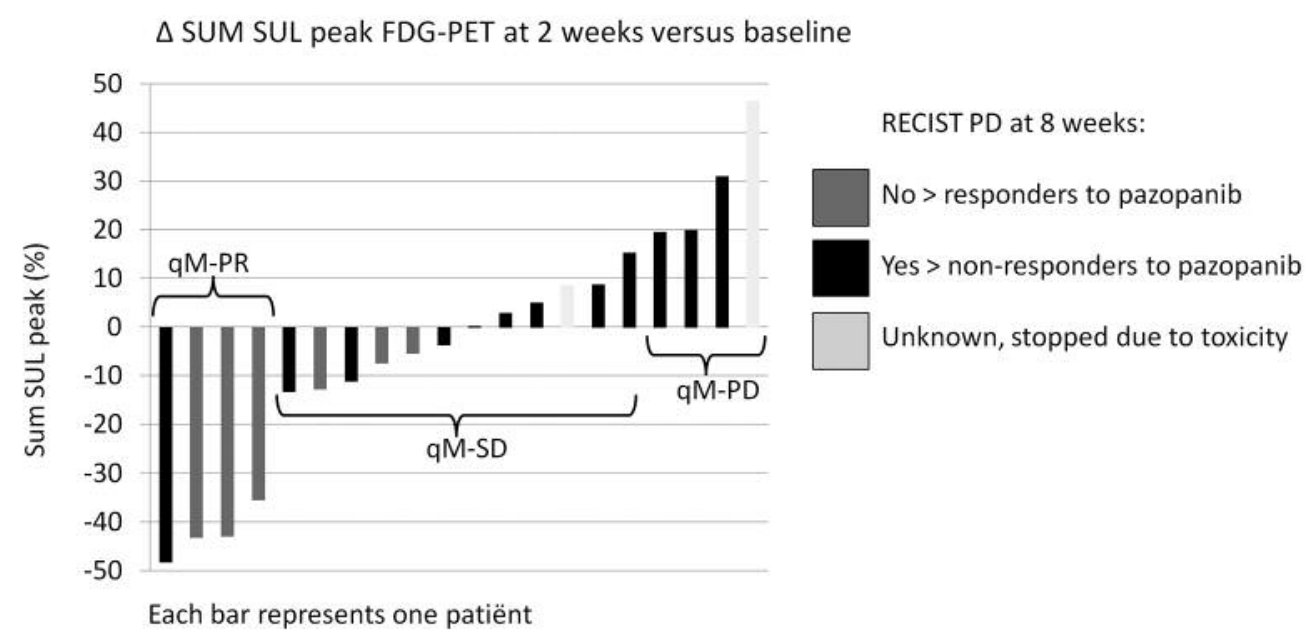

\begin{tabular}{|c|c|c|c|c|}
\hline & & & & \\
\hline & & Responders & Non-responders & Total $(n=18)$ \\
\hline \multirow{3}{*}{ FDG-PET $\Delta$ sum SUL peak at 2 weeks vs baseline } & $\mathrm{qM}-\mathrm{PR}$ & 3 & 1 & 4 \\
\hline & qM-SD & 3 & 8 & 11 \\
\hline & qM-PD & 0 & 3 & 3 \\
\hline \multirow{3}{*}{ FDG-PET $\Delta$ sum SUL peak at 8 weeks vs baseline } & qM-PR & 3 & 0 & 3 \\
\hline & qM-SD & 2 & 5 & 7 \\
\hline & qM-PD & 0 & 3 & 3 \\
\hline \multirow{3}{*}{$\begin{array}{l}\text { Visual analysis by a nuclear clinician of } \\
\text { FDG-PET at } 2 \text { weeks vs baseline }\end{array}$} & visual-PR & 5 & 4 & 9 \\
\hline & visual-SD & 1 & 3 & 4 \\
\hline & visual-PD & 0 & 5 & 5 \\
\hline
\end{tabular}

Figure 3. FDG-PET response compared to clinical outcome. All patients who showed any degree of increase in metabolic activity ( $n=10,0.25 \%$ $46.47 \%$ ) at two weeks, excluding the two who stopped prematurely due to toxicity, were radiologically confirmed as non-responders after eight weeks of therapy.

increase in FDG uptake, whereas the other tissue sites showed an early decrease in FDG uptake after two weeks of therapy (Figure 4). A high or low maximum SUL peak at baseline did not correlate to a difference in PFS and due to the small size of the study group, differences in metabolic responses between tumor histology could not be analyzed.

Pharmacokinetics. All 20 patients completed the PK assessment after two weeks of pazopanib therapy, and 13 patients completed the second PK assessment after eight weeks (which are the same patients who completed the FDG$\mathrm{PET} / \mathrm{CT}$ at eight weeks). Both the $\mathrm{AUC}_{0-24 \mathrm{~h}}$ and $\mathrm{C}_{\text {trough }}$ levels were considered in the analysis. A geometric mean $\mathrm{AUC}_{0-24 \mathrm{~h}}$ (range) of $847 \mu \mathrm{g} . \mathrm{h} / \mathrm{ml}(477-1,654)$ was found after two weeks, and $732 \mu \mathrm{g} . \mathrm{h} / \mathrm{ml}(206-1,264)$ after eight weeks. The median $\mathrm{C}_{\text {trough }}$ level was $26.4 \mu \mathrm{g} / \mathrm{ml}$ (range $=16.6-$ $56.5 \mu \mathrm{g} / \mathrm{ml}$ ) and $33.3 \mu \mathrm{g} / \mathrm{ml}$ (range $=8.0-45.4 \mu \mathrm{g} / \mathrm{ml}$ ) at two and eight weeks, respectively. $\mathrm{AUC}_{0-24 \mathrm{~h}}$ data were significantly correlated with Ctrough levels at both assessments (Spearman rho 0.96 and $0.97, p<0.001)$. Early metabolic response at two weeks was compared to pazopanib exposure at two weeks. No correlation was seen between the quantitative FDG response and $\mathrm{AUC}_{0-24 \mathrm{~h}}$ or $\mathrm{C}_{\text {trough }}$ levels (data not shown) or between exposure and treatment response (Figure 5).

\section{Discussion}

Metastatic STS is a rare group of tumors for which there are few therapeutic options. Unfortunately, a large proportion of STS patients treated with pazopanib, the first available oral targeted drug for this patient group, will not respond to therapy, but may experience serious toxicity $(2,3)$. This underpins the need for a biomarker to identify nonresponders in an early stage. This study evaluated the value of metabolic response assessment by FDG-PET/CT for determining pazopanib treatment efficacy in STS patients 


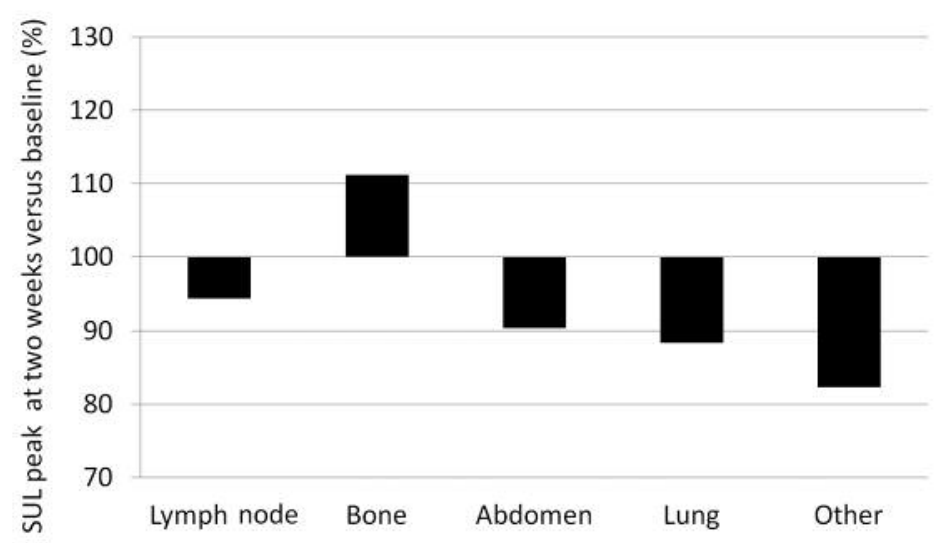

\begin{tabular}{|c|c|c|}
\hline Subtype & N & Range \\
\hline Lymphnode & 12 & $61-124$ \\
\hline Bone & 8 & $77-150$ \\
\hline Abdomen & 14 & $53-195$ \\
\hline Lung & 12 & $38-135$ \\
\hline Other & 6 & $57-118$ \\
\hline
\end{tabular}

Figure 4. Geometric mean of the $\triangle S U L$ per metastatic site at FDG-PET at two weeks versus baseline. Of the eight patients with bone lesions five were non-responders, one patient responded to pazopanib treatment, and two patients discontinued pazopanib prematurely due to toxicity.

and showed promising results. The two weeks' time point for evaluation was chosen, as pazopanib reached a steady state by then and thereby hypothetically represents the optimal metabolic response. Early metabolic progression at two weeks was only seen in RECIST non-responders at eight weeks, and is therefore a potential indication for early discontinuation of pazopanib treatment. As FDG-PET/CT scanning is widely accessible, non-invasive, and the costs could be compensated by the reduction in pazopanib administration for several weeks, this diagnostic method has potentially clinical value.

Overall, metabolic progressive disease at two weeks was a good predictor of non-response to pazopanib according to RECIST criteria at eight weeks. When using the criterion for metabolic progressive disease at two weeks to identify nonresponders, it would reduce treatment beyond the two weeks in $25 \%$ of the patients ( 3 of 12) as assessed by quantitative PERCIST analysis and in $42 \%$ (5 of 12) as evaluated by visual analysis, without false discontinuation. As visual analysis of FDG-PET/CT identified more non-responders than the laborious quantitative analysis and is much easier to perform in a routine clinical practice, visual analysis is preferable (20).

Importantly, in this study population, bone lesions had the largest increase in FDG uptake two weeks after the initiation of treatment compared to baseline. A possible explanation could be the flare response phenomenon, which may be explained by e.g. activation of inflammatory cells or an increase in osteoblastic activity at the site of metastatic bone lesions due to the rapid repair, and thus represents response to treatment rather than disease progression. The flare response is a well-known phenomenon and has been described in the literature for various treatments, including anti-angiogenic therapy for bone metastases in, among others, NSCLC (21). However, the hypothesis of limited pazopanib exposure due to an impaired blood circulation in bone lesions could suggest the exact opposite. When analyzing bone lesions, it is important to be aware of this unexplained phenomenon, especially as radiological response in bone lesions without a clear soft tissue component is difficult by RECIST (22).

In addition to the studies with FDG-PET/CT, the association between FDG uptake and pazopanib exposure was studied. FDG uptake seems to be an independent biomarker of pazopanib treatment response, as variability of the pharmacokinetics of pazopanib did not influence FDG response.

As this is an exploratory study, some limitations need to be addressed. Firstly, our study population had a relatively high number of non-responders $(60 \%, 12$ of 20), when compared to the $23 \%$ non-responders in a phase 3 study (2). As we used similar inclusion and exclusion criteria, this is most likely explained by variations due to our small sample size. Secondly, as metastases can be relatively small and some STS subtypes are known for their indolent growth and relatively low metabolism, FDG-PET/CT evaluation may be less suitable in those STS patients.

In conclusion, this study successfully evaluated the clinically relevant hypothesis of using FDG-PET/CT as an early biomarker of pazopanib treatment in STS patients. We demonstrated that metabolic progression on FDG-PET/CT two weeks after the start of pazopanib can predict progression of disease according to RECIST, assessed 6 weeks later. In this small heterogeneous sarcoma cohort there was no relation between pazopanib exposure and FDG response, suggesting that FDG could be an independent early biomarker of pazopanib failure. Based on our results, a larger study can be set up to validate implementation of FDG- 

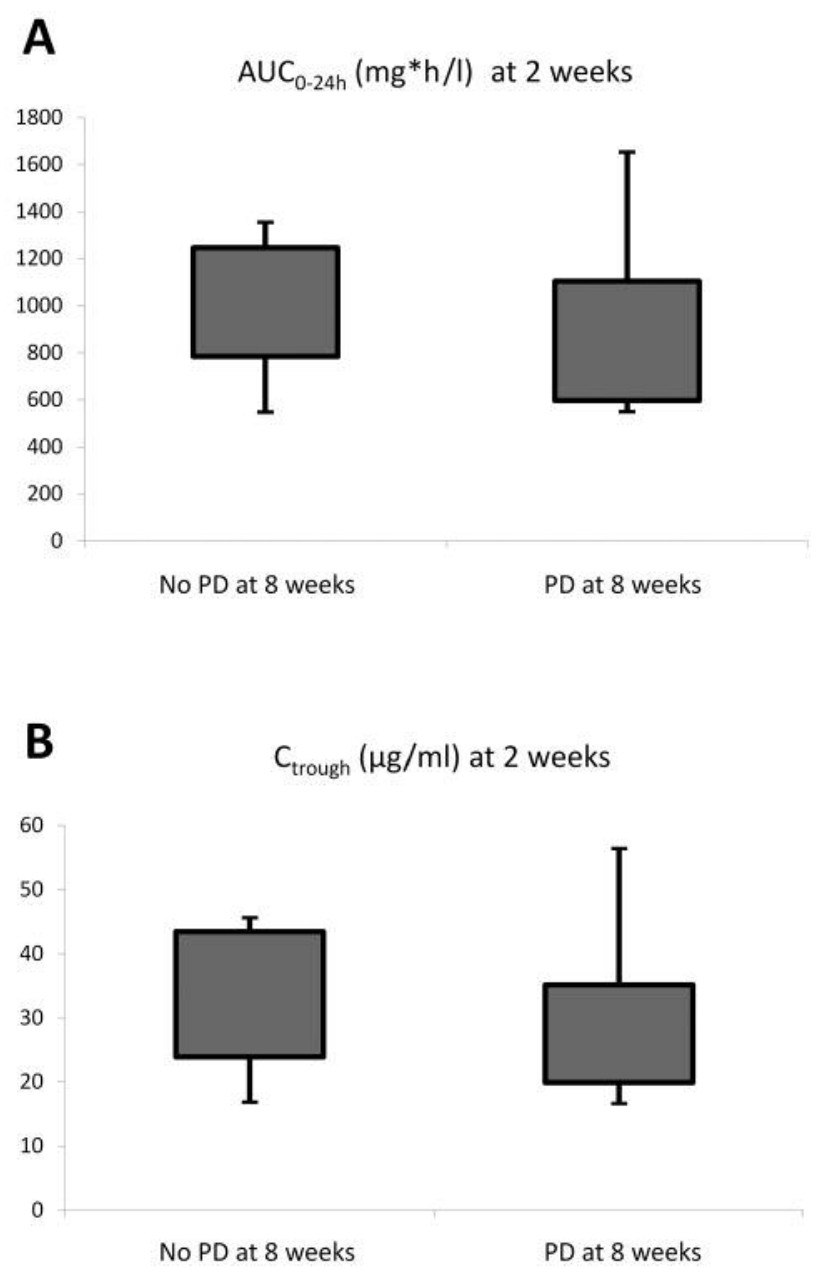

Figure 5. Association between pazopanib pharmacokinetics and response at eight weeks.

PET/CT in the early evaluation of the response in STS patients to pazopanib.

\section{Conflicts of Interest}

Winette van der Graaf and Nielka van Erp received an institutional research Grant from Novartis. As of November 2017, Remy Verheijen is an employee of AstraZeneca, Cambridge, U.K.

\section{Authors' Contributions}

WO, NvE and WvdG designed the study. MV, NS, ID AMK and WvdG were responsible for patient inclusion and acquisition of the data. MV, WO, WG, LFdGO analyzed and interpreted the FDG-PET data. MV, RV, NvE performed the analysis and interpretation of the PK data. MV, WO, NS, ID, RV, AMK, WG, LFdGO, NvE and WvdG were involved in writing and revising the manuscript. All authors gave their final approval of the manuscript.

\section{Acknowledgements}

This academic investigator-initiated study was supported by a grant from GlaxoSmithKline/ Novartis.

\section{References}

1 Fletcher CD, Hogendoorn P, Mertens F and Bridge J: WHO Classification of Tumours of Soft Tissue and Bone. Lyon, France: IARC Press, 2013.

2 van der Graaf WT, Blay JY, Chawla SP, Kim DW, Bui-Nguyen B, Casali PG, Schoffski P, Aglietta M, Staddon AP, Beppu Y, Le Cesne A, Gelderblom H, Judson IR, Araki N, Ouali M, Marreaud S, Hodge R, Dewji MR, Coens C, Demetri GD, Fletcher CD, Dei Tos AP and Hohenberger P: EORTC Soft Tissue and Bone Sarcoma Group: Pazopanib for metastatic softtissue sarcoma (PALETTE): a randomised, double-blind, placebo-controlled phase 3 trial. Lancet 379: 1879-1886, 2012. PMID: 22595799. DOI: 10.1016/S0140-6736(12)60651-5.

3 Sleijfer S, Ray-Coquard I, Papai Z, Le Cesne A, Scurr M, Schoffski P, Collin F, Pandite L, Marreaud S, De Brauwer A, van Glabbeke M, Verweij J and Blay JY: Pazopanib, a multikinase angiogenesis inhibitor, in patients with relapsed or refractory advanced soft tissue sarcoma: a phase II study from the European organisation for research and treatment of cancer-soft tissue and bone sarcoma group (EORTC study 62043). J Clin Oncol 27: 3126-3132, 2009. PMID: 19451427, DOI: 10.1200/JCO.2008.21.3223.

4 Dimitrakopoulou-Strauss A, Strauss LG, Schwarzbach M, Burger C, Heichel T, Willeke F, Mechtersheimer G and Lehnert T: Dynamic PET 18F-FDG studies in patients with primary and recurrent soft-tissue sarcomas: impact on diagnosis and correlation with grading. J Nucl Med 42: 713-720, 2001. PMID: 11337565.

5 Herrmann K, Benz MR, Czernin J, Allen-Auerbach MS, Tap WD, Dry SM, Schuster T, Eckardt JJ, Phelps ME, Weber WA and Eilber FC: 18F-FDG-PET/CT Imaging as an early survival predictor in patients with primary high-grade soft tissue sarcomas undergoing neoadjuvant therapy. Clin Cancer Res 18: 2024-2031, 2012. PMID: 22338012, DOI: 10.1158/1078-0432.

6 Tateishi U, Kawai A, Chuman H, Nakatani F, Beppu Y, Seki K, Miyake M, Terauchi T, Moriyama N and Kim EE: PET/CT allows stratification of responders to neoadjuvant chemotherapy for highgrade sarcoma: a prospective study. Clin Nucl Med 36: 526-532, 2011. PMID: 21637052, DOI: 10.1097/RLU.0b013e3182175856.

7 Jager PL, Gietema JA and van der Graaf WT: Imatinib mesylate for the treatment of gastrointestinal stromal tumours: best monitored with FDG PET. Nucl Med Commun 25: 433-438, 2004. PMID: 15100500.

8 Stroobants S, Goeminne J, Seegers M, Dimitrijevic S, Dupont P, Nuyts J, Martens M, van den Borne B, Cole P, Sciot R, Dumez $\mathrm{H}$, Silberman S, Mortelmans L and van Oosterom A: ${ }^{18}$ FDGPositron emission tomography for the early prediction of response in advanced soft tissue sarcoma treated with imatinib mesylate (Glivec). Eur J Cancer 39: 2012-2020, 2003. PMID: 12957455.

9 Farnebo J, Gryback P, Harmenberg U, Laurell A, Wersall P, Blomqvist LK, Ullen A and Sandstrom P: Volumetric FDG-PET predicts overall and progression-free survival after 14 days of targeted therapy in metastatic renal cell carcinoma. BMC Cancer 14: 408, 2014. PMCID: PMCPMC4064288, DOI: 10.1186/14712407-14-408. 
10 Verheijen RB, Yu H, Schellens JHM, Beijnen JH, Steeghs N and Huitema ADR: Practical Recommendations for Therapeutic Drug Monitoring of Kinase Inhibitors in Oncology. Clin Pharmacol Ther, 2017. PMID: 28699160, DOI: 10.1002/cpt.787.

11 Suttle AB, Ball HA, Molimard M, Hutson TE, Carpenter C, Rajagopalan D, Lin Y, Swann S, Amado R and Pandite L: Relationships between pazopanib exposure and clinical safety and efficacy in patients with advanced renal cell carcinoma. $\mathrm{Br}$ J Cancer 111: 1909-1916, 2014. PMID: 25349968, DOI: 10.1038/bjc.2014.503.

12 Verheijen RB, Swart LE, Beijnen JH, Schellens JHM, Huitema ADR and Steeghs N: Exposure-survival analyses of pazopanib in renal cell carcinoma and soft tissue sarcoma patients: opportunities for dose optimization. Cancer Chemother Pharmacol 80: 1171-1178, 2017. PMID: 29051995, DOI: 10.1007/s00280-017-3463-x.

13 Bellesoeur A, Boudou-Rouquette P, Thomas-Schoemann A, Joly C, Tlemsani C, Vidal M, Goldwasser F and Blanchet B: Individualized pazopanib dosing-letter. Clin Cancer Res 23: 6377, 2017. PMID: 29030334, DOI: 10.1158/1078-0432.

14 Eisenhauer EA, Therasse P, Bogaerts J, Schwartz LH, Sargent D, Ford R, Dancey J, Arbuck S, Gwyther S, Mooney M, Rubinstein L, Shankar L, Dodd L, Kaplan R, Lacombe D and Verweij J: New response evaluation criteria in solid tumours: revised RECIST guideline (version 1.1). Eur J Cancer 45: 228247, 2009. PMID: 19097774, DOI: 10.1016/j.ejca.2008.10.026.

15 National Cancer Institute. Common Terminology Criteria for Adverse Events v.4.0 (CTCAE).

16 Boellaard R, Delgado-Bolton R, Oyen WJ, Giammarile F, Tatsch $\mathrm{K}$, Eschner W, Verzijlbergen FJ, Barrington SF, Pike LC, Weber WA, Stroobants S, Delbeke D, Donohoe KJ, Holbrook S, Graham MM, Testanera G, Hoekstra OS, Zijlstra J, Visser E, Hoekstra CJ, Pruim J, Willemsen A, Arends B, Kotzerke J, Bockisch A, Beyer T, Chiti A and Krause BJ; European Association of Nuclear M: FDG PET/CT: EANM procedure guidelines for tumour imaging: version 2.0. Eur J Nucl Med Mol Imaging 42: 328-354, 2015. PMID: 25452219, DOI: 10.1007/s00259-014-2961-x.
17 Wahl RL, Jacene H, Kasamon Y and Lodge MA: From RECIST to PERCIST: Evolving Considerations for PET response criteria in solid tumors. J Nucl Med 50: 122S-150S, 2009. PMID: 19403881, DOI: 10.2967/jnumed.108.057307.

18 Janmahasatian S, Duffull SB, Ash S, Ward LC, Byrne NM and Green B: Quantification of lean bodyweight. Clin Pharmacokinet 44: 1051-1065, 2005. PMID: 16176118, DOI: 10.2165/ 00003088-200544100-00004.

19 van Erp NP, de Wit D, Guchelaar HJ, Gelderblom H, Hessing TJ and Hartigh J: A validated assay for the simultaneous quantification of six tyrosine kinase inhibitors and two active metabolites in human serum using liquid chromatography coupled with tandem mass spectrometry. J Chromatogr B Analyt Technol Biomed Life Sci 937: 33-43, 2013. PMID: 24013127 , DOI: 10.1016/j.jchromb.2013.08.013.

20 Willemsen AE, Vlenterie M, van Herpen CM, van Erp NP, van der Graaf WT, de Geus-Oei LF and Oyen WJ: Positron emission tomography response criteria in solid tumours criteria for quantitative analysis of (18F)-fluorodeoxyglucose positron emission tomography with integrated computed tomography for treatment response assessment in metastasised solid tumours: All that glitters is not gold. Eur J Cancer 56: 54-58, 2016. PMID: 26808297, DOI: 10.1016/j.ejca.2015.12.017.

21 Krupitskaya Y, Eslamy HK, Nguyen DD, Kumar A and Wakelee HA: Osteoblastic bone flare on F18-FDG PET in non-small cell lung cancer (NSCLC) patients receiving bevacizumab in addition to standard chemotherapy. J Thorac Oncol 4: 429-431, 2009. PMID: 19247091, DOI: 10.1097/JTO.0b013e3181989e12.

22 Costelloe CM, Chuang HH, Madewell JE and Ueno NT: Cancer Response Criteria and Bone Metastases: RECIST 1.1, MDA and PERCIST. J Cancer 1: 80-92, 2010. PMID: 20842228.

Received January 6, 2019

Revised January 19, 2019

Accepted January 22, 2019 